\title{
IFH山
}

\section{WIKIVANTES: ¿UN PROYECTO DE HUMANIDADES DIGITALES?}

\section{THE WIKIVANTES: A DIGITAL HUMANITIES PROJECT?}

Francisco Javier Bravo Ramón

javierbravoramon@gmail.com

\section{Resumen}

La wikivantes (http://wikivantes. wikispaces.com) surgió con la idea de utilizar la obra completa de Miguel de Cervantes, los aspectos de su vida y el entorno histórico como modelo educacional, a partir de fragmentos significativos que se pudieran enlazar de forma hipertextual con diversas disciplinas. El proyecto se basa en los cuatro pilares de la educación del informe Delors, La educación encierra un tesoro: Aprender a aprender, aprender a hacer, aprender a vivir con los demás y aprender a ser, con la intención de crear un repositorio de recursos, aplicaciones e ideas que contribuyan a mejorar la sociedad del futuro desde los textos cervantinos. La pregunta que nos formulamos en este artículo es si un proyecto como la wikivantes se puede considerar como perteneciente al ámbito de las Humanidades Digitales.

Palabras clave: Cervantes. Wiki. Humanidades Digitales. Educación. Informe Delors.

\begin{abstract}
The wikivantes (http://wikivantes.wikispaces.com) arose with the goal of making use of Cervantes work, and some facets of his life and historic environment, as educational paradigm, from meaningful passages which could be connected as hypertext with several disciplines. The project is based on the four pillars of learning from Delors report, La educación encierra un tesoro: Learning to know, learning to do, learning to live together and learning to be; with the aim to make a repository of resources, applications and ideas that contribute to improve
\end{abstract}


the society of the future with Cervantes work. The query we set out in this paper is if the wikivantes can be considered as a Digital Humanities Project.

Keywords: Cervantes. Wiki. Digital Humanities. Education. Delors report.

\section{INTRODUCCIÓN}

La wikivantes ${ }^{1}$ surgió inicialmente como un proyecto de innovación educativa para trabajar las competencias básicas de la enseñanza media en España, a partir de los cuatro pilares de la educación mencionados en el informe Delors. A estas competencias básicas o clave (ocho inicialmente, siete en aquellas comunidades en las que se comenzó a implantar la LOMCE, Ley Orgánica para la Mejora de la Calidad Educativa) se le unía una novena (u octava) que se denominó competencia cervantina y que tenía un carácter transversal, multidisciplinar, abierto y colaborativo.

Tras su diseño inicial, la wikivantes abandonó el concepto basilar de las competencias básicas, aunque se siguiera contemplando su aplicación y su transformación en competencias profesionales, entendidas como habilidades, y se orientó a un tratamiento medular, a partir de los cuatro pilares del aprendizaje: el aprender a conocer, aprender a hacer, aprender a ser y aprender a vivir con los demás.

En cada uno de estos módulos se ofrecían, y así se continúa haciendo, una serie de invitaciones a abordar los textos cervantinos desde perspectivas, disciplinas y planteamientos tan diversos como propuestas pudieran surgir, de forma individual o colectiva, local o global, con afán abarcador o limitado a un módulo único o a un proyecto puntual.

La base formal del proyecto utilizaba las posibilidades que ofrecen las wikis para realizar un trabajo abierto y colaborativo en un entorno educativo no restrictivo ni excluyente. No se ofrecían, ni tampoco se hace en la actualidad, recursos originales, ni aplicaciones para implementar tal o cual contenido. Se utilizaban algunas de las existentes (webquest, cazas del tesoro, grabación de vídeos y audios) para mostrar, a modo de ejemplo, una forma concreta de trabajar los textos cervantinos propuestos que, a su vez, eran susceptibles de abordarse, los mismos fragmentos $u$ otros, con múltiples recursos y aplicaciones y desde disciplinas y perspectivas diversas.

El objetivo principal de la wikivantes tenía, y sigue teniendo, la intención de convertirse en un soporte educativo y socio-cultural que, partiendo de los textos cervantinos y de la propia

\footnotetext{
${ }^{1}$ Accesible desde http://wikivantes.wikispaces.com/.
} 
vida del escritor alcalaíno, y lejos del sentido enciclopedista de otros planteamientos que priman los contenidos, pudiera convertirse en un método práctico de aprendizaje holístico en el que se recogieran experiencias sobre el saber, el hacer, el ser y el convivir, con el paradigma de lo cervantino como punto de partida, llegada o simplemente de acompañamiento en el camino o viaje.

\section{CERVANTES COMO EDUCADOR}

En la mayoría de las culturas podemos encontrar grandes nombres, devenidos en clásicos, que se convirtieron en educadores de sus respectivos pueblos. Esta tradición comenzó en Occidente, en Grecia a través de Homero, como bien señaló Marrou (1970: 26) recogiendo las opiniones y comentarios de la Antigüedad:

La educación literaria griega, a lo largo de toda su historia, conservó a Homero como texto básico, como centro de todos los estudios [...] Como lo dijo Platón, Homero fue, en el cabal sentido de la palabra, el educador de Grecia, ten Hellada pepáideuken.

Los textos de los grandes escritores y pensadores europeos han servido, en mayor o menor medida, de educadores escolares, pero también, se han convertido en formadores de un espíritu que ha trascendido la enseñanza para convertirse en la base de la cultura sobre la que se sustenta lo universal. $Y$ en eso radica una de sus grandezas, como también señalaba Marrou (1970: 31):

La paideia ya no es solamente la técnica apropiada para el niño, pais, que lo equipa, lo prepara precozmente para que se convierta en hombre; merced a una notable amplificación, áuxesis, el mismo vocablo, en el griego helenístico, sirve para designar el resultado de este esfuerzo educativo, proseguido más allá de la escuela, durante toda la vida, con el objeto de realizar con mayor perfección el ideal humano. La paideia (o paideusis) llega a significar la cultura, concebida no en el sentido activo, preparatorio, de la educación, sino en el sentido perfectivo que esta voz tiene hoy día entre nosotros: el estado de un espíritu plenamente desarrollado, en el cual han florecido todas sus virtualidades, el del hombre que ha llegado a ser verdaderamente hombre. Y es digno de señalarse que Varrón y Cicerón, cuando deban traducir paideia, escogerán, en latín, la palabra humanitas.

La elección de los autores clásicos como educadores se nos antoja irrebatible, dada su cualidad de obras, y por extensión de enseñanzas, de primer orden, como apunta García Gual (2005: 26):

Las obras y autores que la tradición ha estimado como dignos de perdurar son, esencialmente, los calificados 'clásicos'. Esas obras 'de primera clase' son las que 
parecen ofrecer una lección perdurable, las que, por así decir, se definen como paradigmáticas e inagotables $y$, diríamos, parecen poder releerse infinitamente.

Nos interesa incidir en dos términos referidos a los textos clásicos del fragmento de García Gual: paradigmáticas, es decir aquellas obras susceptibles de imitar por ser un ejemplo; e inagotables, o lo que es lo mismo, revisables y utilizables a lo largo del tiempo, tanto en su interpretación sincrónica como en su devenir diacrónico.

Nadie pone en duda que Miguel de Cervantes es el gran clásico de la literatura, y por extensión, de la cultura española, y quizá más aún, su inmortal personaje que trascendió el puro nominalismo, para adquirir un carácter semántico pleno, con las acepciones que todos conocemos. Pero lo que nos planteamos aquí, es su pertinencia y actualidad como educador de la sociedad de hoy en día y referente de las Humanidades en un sentido amplio. Nos parece muy acertada, al hilo de lo apuntado, la lectura que hace María González (2009: 1314) sobre la necesidad de comprender el Quijote desde una perspectiva de invención activa:

De la mano del Quijote, es posible contribuir a la aportación de soluciones para la actual crisis de las Humanidades tejiendo una innovadora teoría de comprensión lectora de los clásicos que va más allá de la típica lectura consumista donde pasivamente se guarda el texto en la memoria y de la lectura analítica donde se trabaja sólo el eco de la intención del autor, pues no sólo nos interesa leer para archivar mensajes o identificar intenciones, sino también para trabajar su sentido en relación con las expectativas significativas y libres del lector a fin de promover su transformación dialógica y creativa en los nuevos contextos receptivos donde el canon vive del hipertexto y la literariedad o condición generativa de la escritura devuelve a la estrategia intertextual su protagonismo inventivo: el autor, ya sea el que escribió o bien el que lee y corresponde, siempre piensa a partir de su comprensión del otro.

El valor de la obra completa de Cervantes trasciende lo puramente literario para convertirse en depositario de una serie de valores cuyas características fundamentales residen en la universalidad de sus ideas y en la transversalidad de tratamientos desde los que se pueden abordar, pues como sostiene también González (2009: 38):

La "vida" de la literatura es un saco cervantino siempre "roto". Luego más que de Literatura o de clásicos específicamente "hispánicos" deberíamos proceder al estudio de una literatura intercultural entre pueblos y artes, tal y como nos muestran aquellas investigaciones que trabajan la intersección estética de tópicos y mitos con el objetivo de desarrollar en el ámbito estudiantil el horizonte de expectativas señalado por Jauss donde se comprende la historia a través del entramado de las manifestaciones artísticas y las ideologías que comportan.

Nuestra propuesta tiene un carácter claramente vindicatorio del papel que deberían ocupar las Humanidades en la educación integral de las personas, desde premisas actuales y en el marco de integración curricular de cualquier actividad, formal o no formal. Pero también 
es un intento de ampliar el campo de acción de lo cervantino, limitado por lo general a lo quijotesco, con el convencimiento de que los principios educativos que se pretenden trabajar no sólo residen en la novela inmortal del alcalaíno, sino en su obra completa.

No obstante, y a pesar de que el proyecto tiene unas bases marcadamente didácticas, parte de una postura irónicamente antipedagógica, utilizando la expresión de González Faraco (2010: 39), para quien:

Una lectura antipedagógica es una lectura gozosa -alegre, triste, dramática, cómicaque busca recuperar y revivir no las palabras sino la pasión y el testimonio radical del escritor, su humanidad, y en ella el sentido profundamente narrativo y misterioso de la experiencia, no siempre amable, del vivir.

Y a través del inagotable ejemplo de los clásicos, y de su capacidad para renovarse como educadores con cada nueva lectura, retornamos al fragmento de Marrou sobre la interpretación latina del término griego paideia, la humanitas, entendida como ese humanismo que el Diccionario de la Lengua Española define como: "Doctrina o actitud vital basada en una concepción integradora de los valores humanos" (DRAE, 2016).

\section{VISIÓN ¿QUIJOTESCA? ACTUAL DE LAS HUMANIDADES}

En un mundo como el actual se impone con mayor sentido el cultivo de la humanidad, aunque para ello se requieran destrezas, que podrán devenir en competencias, básicas o no. Martha C. Nussbaum (2005: 29-31) apunta tres habilidades para cultivar hoy en día la humanidad:

La primera es la habilidad para un examen crítico de uno mismo y de las propias tradiciones, que nos permita experimentar lo que, siguiendo a Sócrates, podríamos llamar 'vida examinada'. Es decir, una vida que no acepta la autoridad de ninguna creencia por el solo hecho de que haya sido transmitida por la tradición o se haya hecho familiar a través de la costumbre; una vida que cuestiona todas las creencias y sólo acepta aquellas que sobreviven a lo que la razón exige en cuanto a coherencia y justificación. Esta disciplina requiere el desarrollo de la habilidad de razonar lógicamente, de poner a prueba lo que uno lee o dice desde el punto de vista de la solidez del razonamiento, de la exactitud de los hechos y la precisión del juicio. [...] Los ciudadanos que cultivan su humanidad necesitan, además, la capacidad de verse a sí mismos no sólo como ciudadanos pertenecientes a alguna región o grupo, sino también, y sobre todo, como seres humanos vinculados a los demás seres humanos por lazos de reconocimiento y mutua preocupación. El mundo a nuestro alrededor es ineludiblemente internacional. [...] Pero los ciudadanos no pueden reflexionar bien sobre la sola base del conocimiento factual. La tercera destreza que debe poseer el ciudadano, estrechamente relacionada con las dos primeras, se puede llamar imaginación narrativa. Esto significa la capacidad de pensar cómo sería estar en el lugar de otra persona, ser un lector inteligente de la historia de esa persona, $y$ comprender las emociones, deseos y anhelos que alguien así pudiera experimentar. 
Conceptos muy interesantes los apuntados por Nussbaum, y sobre los que podríamos volver una y otra vez desde los textos cervantinos, para realizar una vindicación necesaria de las Humanidades, en estos tiempos en los que parece que en la aldea global hay una idea un tanto confusa de lo universal, que quizá sea necesario reformular, pues como dice Philippe Meirieu (2001: 45):

Sin duda, todo educador es portador de valores. $Y$, sin duda, la afirmación misma de un valor implica cierta pretensión de universalidad. En efecto, ¿qué es lo que 'vale algo' hasta el punto de querer compartirlo con los demás si no es, precisamente, aquello que considero que va más allá de mi interés personal, de mis necesidades del momento, de mi situación no esencial y provisional? Si existe un valor que la actividad educativa nos hace ver, es la misma exigencia del valor, de algo que merece que eduquemos con ello a las personas, de algo que ya nadie se atreve a llamar 'el bien', pero que se sigue postulando, cada vez que sugerimos $-y$ lo hacemos constantemente- dónde se encuentra 'lo mejor'.

La enseñanza de lo universal como valor de la sociedad de la información, devenida en sociedad del conocimiento, debe, no obstante, complementarse con otro gran concepto, quizá uno de los más cervantinos entre tantos otros, el de la libertad. Y nadie como Fernando Savater (2001) explica la esencia de la libertad en términos educativos:

El objetivo explícito de la enseñanza en la modernidad es conseguir individuos auténticamente libres. [...] La libertad de la que estamos hablando no es un a priori ontológico de la condición humana sino un logro de nuestra integración social. A ello apuntaba Hegel, cuando estableció que 'ser libre no es nada, devenir libre lo es todo'. No partimos de la libertad, sino que llegamos a ella. Ser libre es liberarse: de la ignorancia prístina, del exclusivo determinismo genético moldeado según nuestro entorno natural y/o social, de apetitos e impulsos instintivos que la convivencia enseña a controlar.

Y es también Savater el que nos facilita las claves sobre las que gravitará nuestro proyecto innovador cervantino, al trascender la libertad desde planteamientos sociales y transformarla en democracia, para así unir el concepto al de lo universal en un sintagma que se constituye en la base de nuestro proceso de enseñanza-aprendizaje: la universalidad democrática. Veamos cómo lo explica

El ideal básico que la educación actual debe conservar y promocionar es la universalidad democrática. [...] Empecemos por la universalidad. ¿Universalidad en la educación? Significa poner al hecho humano-lingüístico, racional, artístico-por encima de sus modismos; valorarlo en su conjunto antes de comenzar a resaltar sus peculiaridades locales; $y$ sobre todo no excluir a nadie a priori del proceso educativo que lo potencia y desarrolla (Savater, 2001). 
Por lo que respecta al concepto democrático en su aplicación educativa y su aportación al sintagma referido, Savater apunta los siguientes componentes que lo conforman:

Asimilación del ingrediente de relatividad que toda política democrática conlleva; fomentar la capacidad de crítica y selección; valorar positivamente la existencia de pluralismo social, así como el conflicto, que no sólo es necesario sino fructífero; estimular la participación en la gestión pública; desarrollar la conciencia de la responsabilidad de cada cual y también del necesario control sobre los representantes políticos; reforzar el diálogo frente al monólogo, el perfil de los discrepantes como rivales ideológicos pero no como enemigos civiles, y aceptar que 'todo el mundo tiene derecho a equivocarse pero nadie posee el de exterminar el error'.

\section{LO DIGITAL-CERVANTINO}

La presencia de lo cervantino en el mundo digital ha sido una constante desde la irrupción de la informática en nuestras vidas de un modo que hoy en día podríamos denominar como integral y prácticamente inevitable, con visos de dependencia en muchos aspectos del quehacer cotidiano ${ }^{2}$.

Esta presencia se materializó en diversos ámbitos diferenciados y no siempre interconectados. En primer lugar, a través de la propia obra cervantina, sometida a digitalizaciones integrales como parciales, gracias a proyectos editoriales implementados en los últimos treinta años, desde instituciones y organismos nacionales e internacionales. En segundo término, habría que destacar el acceso disponible a una ingente bibliografía cervantina y a estudios críticos de diversas procedencias y épocas ${ }^{3}$. Ha sido importante asimismo la creación de portales virtuales para conmemorar algunas efemérides cervantinas, que han servido para unificar y reunir recursos de gran interés ${ }^{4}$. También son de destacar los proyectos de investigación ligados a universidades españolas o extranjeras, que han centrado sus labores digitales en aspectos globales o parciales de la producción cervantina ${ }^{5}$.

\footnotetext{
${ }^{2}$ Sería muy extenso enumerar todos los recursos sobre Cervantes disponibles en la red, por lo que nos limitaremos a remitir al portal del autor en la biblioteca virtual que lleva su nombre:

http://www.cervantesvirtual.com/portales/miguel de cervantes/.

${ }^{3}$ Además del acceso que facilita la página de autor del Cervantes Virtual, habría que mencionar la labor que desde hace años se viene realizando en el Centro de Estudios Cervantinos (CEC), http://www.centroestudioscervantinos.es/index.php?itm=2.1 de Alcalá de Henares, las actas de los diversos congresos cervantistas celebrados en los últimos años (disponibles en formato electrónico) o la existencia de revistas digitales cervantinas, como la pionera Anales Cervantinos, http://analescervantinos.revistas.csic.es/index.php/analescervantinos, 0 eHumanista Cervantes http://www.ehumanista.ucsb.edu/cervantes de creación más reciente.

${ }^{4}$ El ejemplo más notable y actual es la creación por parte de la BNE (Biblioteca Nacional de España) de un espacio virtual para conmemorar el IV Centenario de la muerte de Miguel de Cervantes, http://cervantes.bne.es, que engloba multitud de recursos de diverso tipo. O la página web del Ministerio de Educación creada para celebrar tal evento: http://400cervantes.es/.

${ }^{5}$ Uno de los proyectos pioneros en el ámbito de las Humanidades Digitales fue la edición del Quijote Variorum http://archive.is/cervantes.tamu.edu, Ilevada a cabo por Eduardo Urbina en la Universidad A\&M de Texas en colaboración con la cátedra Cervantes de la UCLM, que reunió multitud de recursos cervantinos, pero que no ha tenido la continuidad deseada, pues los enlaces no se han actualizado y los dominios no se han mantenido.
} 
Otra cuestión sería determinar cuántos y cuáles de todos los proyectos existentes sobre Cervantes, aun siendo digitales o electrónicos en muchos casos, se pueden encuadrar en el ámbito metodológico de las Humanidades Digitales. Por poner un ejemplo, los recursos disponibles, cervantinos o no, en el Cervantes Virtual 6 ofrecen un enlace en HTML, pero no en lenguaje de marcado XML, aunque se ha incorporado recientemente un acceso a los datos y metadatos de la web semántica de cada entrada, que permite la descarga en RDF, JSON, Dublin Core y ESE. ¿Se pueden enmarcar estos recursos en el campo de las Humanidades Digitales?

\section{LA WIKIVANTES COMO PROYECTO SOCIO-CULTURAL Y EDUCATIVO}

\subsection{Conceptualización}

Como ya anticipamos en la introducción, la wikivantes es una propuesta de carácter educativo, social y cultural para construir una sociedad libre, moderna y solidaria a partir de los textos de Miguel de Cervantes. En ella se parte de una serie de fragmentos significativos de distintas obras cervantinas que permitan trabajar aspectos diversos, desde un punto de vista literario y textual, didáctico y formativo, cultural y antropológico, y humanístico y social.

La propuesta de trabajo parte de un esquema abierto, que se podrá implementar de forma modular o con carácter integral, aplicado a ámbitos educativos como la ESO y el Bachillerato, grados universitarios, enseñanza no reglada, proyectos culturales de carácter multidisciplinar o cualquier enfoque relacionado con las Humanidades Digitales.

Es ante todo un proyecto colaborativo que tiene en los textos de Cervantes el núcleo a partir del cual se puedan construir los cuatro pilares de la educación mencionados en el informe Delors, La educación encierra un tesoro (1996), y que se definen de la siguiente manera:

Para cumplir el conjunto de las misiones que les son propias, la educación debe estructurarse en torno a cuatro aprendizajes fundamentales que en el transcurso de la vida serán para cada persona, en cierto sentido, los pilares del conocimiento: aprender a conocer, es decir, adquirir los instrumentos de la comprensión; aprender a hacer, para poder influir sobre el propio entorno; aprender a vivir juntos, para participar $y$ cooperar con los demás en todas las actividades humanas; por último, aprender a ser, un proceso fundamental que recoge elementos de los tres anteriores. Por supuesto, estas cuatro vías del saber convergen en una sola, ya que hay entre ellas múltiples puntos de contacto, coincidencia e intercambio.

Centrado en el teatro cervantino está el proyecto de la Universidad de Huelva dirigido por Luis Canseco: http://www.uhu.es/cervantesteatro/proyecto datos.php.

${ }^{6}$ Accesible desde http://www.cervantesvirtual.com/. 
El espíritu que lo sustenta es el mismo del que partía la web 2.0, pero enriquecido con la 3.0, en la que lo relevante parte de los datos, metadatos y de las relaciones que se establezcan entre los enlaces significativos de los temas que se desarrollen, enriquecidas por las aportaciones multidisciplinares que se puedan establecer desde las variadas perspectivas que permite la obra de Cervantes, y con el objetivo central de construir la sociedad del futuro sobre los principios de tolerancia, libertad y pensamiento crítico y creativo.

La fundamentación teórica de la wikivantes tiene muy presentes las palabras de Zhou Nan-Zhao, que al referirse a la metodología para aplicar los cuatro pilares de la educación señala lo siguiente:

To redesign learning modules or units, or curriculum blocks', which teachers and learners could use in light of the nationally set curriculum standards. For example, in facilitating 'learning to learn', modules or units or even a course could be designed to develop connections between disciplines and enable students to see knowledge as an inter-related coherent whole, as implied in the interpenetration of the four pillars of learning [...] This area of interaction goes beyond traditional study skills and include such competencies inherent in the four pillars of learning: collaboration skills; attitudes toward work; communication; reflection; problem solving and thinking skills; and subject-specific and interdisciplinary conceptual understanding. Learning content and method reorganized in such integrated approach help students develop the range of their capacities, positive attitudes and effective habits of mind and should lie at the core of all curriculum development and delivery (Nan-Zhao, 2005).

\subsection{Estructura formal de la propuesta}

La wikivantes se estructura en cuatro módulos correspondientes a cada uno de los aprendizajes señalados, que se desarrollan a su vez en tres bloques temáticos cervantinos:

- Vida y aledaños.

- El Quijote.

- Textos no quijotescos.

Los fragmentos textuales significativos sirven de punto de partida del proyecto y constituyen el eje temático sobre el que se estructura cada uno de los bloques. A partir de cada uno de ellos, se podrán ampliar, si así se desea, las propuestas con nuevos textos, cervantinos o no, de los siglos XVI y XVII o de cualquier época, de contenido literario o con un contenido de otra u otras disciplinas. Tan sólo se fijan un par de normas a la elección de textos complementarios a los textos base cervantinos: que giren en torno a los temas y contenidos tratados en cada bloque y que no tengan una extensión superior a los límites de la lectura hipertextual. 
Cada uno de los cuatro módulos está estructurado en las siguientes categorías relacionadas con Aspectos generales.

- Actividades.

- Programación general.

- Evaluación.

- Textos correspondientes a cada uno de los tres Bloques.

- Propuestas de aprendizaje de los pilares de la educación de cada Módulo.

\subsection{Premisas para su implementación}

Como se apunta en la propia wikivantes, lo que se ofrece es tan solo un punto de partida que pueda servir de pauta a seguir o de la que alejarse, siempre que no desvirtuemos el espíritu de la propuesta. Sería adecuado, por tanto, hablar de las cinco pautas siguientes que posibiliten la implementación del proyecto:

- Se puede partir de los textos cervantinos escogidos o se pueden seleccionar otros distintos.

- Se pueden trabajar algunos de los recursos propuestos o se pueden, y deben, proponer otros.

- Se pueden trabajar algunos de los textos desde módulos o pilares distintos en los que están situados, siempre que se justifique suficientemente la propuesta.

- Se pueden abordar los textos desde disciplinas que no sean necesariamente literarias ni textuales.

- Se pueden relacionar los textos y propuestas con textos y actividades de todo tipo de autores, disciplinas y épocas.

Las pautas señaladas, a su vez, deberían partir de las cinco premisas fundamentales siguientes, en la línea del espíritu del proyecto y en correspondencia con los principios básicos sobre los que se sustentan las Humanidades Digitales:

- Se debe tener una visión abierta, colaborativa y multidisciplinar de los textos y de las Humanidades.

- Se debe trabajar desde planteamientos cercanos a las Humanidades Digitales, con lo que el espíritu de obra abierta, recursos colaborativos y, muy especialmente, el 
enlace de datos deberán ser las premisas conceptuales que guíen cada una de las propuestas.

- Se debe encuadrar cada propuesta, por cuestiones metodológicas y taxonómicas, en uno de los cuatro pilares del aprendizaje, de tal forma que el punto de partida de una aportación deba centrarse de inicio en un tipo concreto de aprendizaje (hacer, conocer, ser o relacionarse con los otros). Esto no implica, necesariamente, que un mismo texto se pueda abordar a posteriori desde los cuatro pilares, de forma transversal, siempre que se justifique suficientemente.

- Se debe primar un acercamiento a los textos y actividades que tenga un carácter global, en el que el auténtico protagonista del aprendizaje sea el proceso en sí, al margen de los resultados que se puedan alcanzar. El resultado es el propio proceso.

- Se debe tender a planteamientos educativos y humanísticos lo menos invasivos posible, con el fin de realizar incursiones laparoscópicas en los textos cervantinos.

\section{6. ¿ES LA WIKIVANTES UN PROYECTO DE HUMANIDADES DIGITALES?}

Decidir si un proyecto como la wikivantes se puede encuadrar en el ámbito de las Humanidades Digitales puede ser muy fácil o muy difícil, dependiendo de los criterios que se utilicen para realizar la evaluación, de la laxitud en la aplicación de los parámetros empleados, del perfil de los responsables encargados del análisis y hasta de los principios y definiciones de las propias Humanidades Digitales como campo disciplinario o como instrumento técnico que facilite el acceso, estudio y difusión de los datos.

No entraremos aquí en definiciones cuasi ontológicas de la disciplina, porque ya se ha escrito bastante en los últimos años sobre ello y porque básicamente no es el tema de este artículo. Tan solo nos gustaría recordar unas palabras de Nuria Rodríguez Ortega, que creemos que dan en la clave del problema:

El uso indiscriminado de la etiqueta 'Humanidades Digitales' para denominar cualquier práctica, iniciativa o proyecto de las disciplinas humanístico-sociales que utilice recursos digitales o tecnologías informáticas me resulta sintomático de una realidad de facto, reconocida por casi todos: en la sociedad del siglo XXI no es posible ya el desarrollo de conocimiento sin contar de algún modo con el factor digital. Por tanto, todo parece susceptible de ser adjetivado como 'digital'. Si a esto le unimos que la sugerencia de modernidad tecnológica que evocan las Humanidades Digitales las ha puesto de moda, tenemos los ingredientes suficientes para que cada vez cosas más diversas y variopintas sean consideradas como tales [...] Contrariamente a lo que una aproximación superficial pudiera hacer pensar, las Humanidades Digitales van más allá de la aplicación y uso de una serie de tecnologías, recursos y sistemas digitales. Lo que define, pues, las Humanidades Digitales frente al conjunto de disciplinas humanísticas que 'utilizan' herramientas tecnológicas es la búsqueda de nuevos modelos interpretativos, nuevos paradigmas disruptivos en la compresión de la cultura 
y del mundo. Corolario lógico: las Humanidades Digitales no implican hacer cosas de modo distinto con la asistencia de la tecnología, sino 'pensar' el mundo de manera diferente a través de las especificidades que definen el medio digital y el pensamiento computacional. Quedarnos en lo primero supondría estar avanzando hacia una tecnologización de las Humanidades, lo cual se encuentra en el polo inverso de lo que, según mi punto de vista, constituye su fundamento real y su relevancia como agente de transformación sociocultural y política: la inclusión crítica del pensamiento humanista en la construcción tecnológica y digital de nuestro mundo. Por tanto, el compromiso de las Humanidades Digitales y, por ende, del humanista digital no se establece con el desarrollo tecnológico, sino con el Hombre, materializando así una vuelta a la esencia del Humanismo (Rodríguez Ortega, 2014: 14-15).

Si hiciéramos una autoevaluación rigurosa para saber si la wikivantes se puede considerar un proyecto de Humanidades Digitales, atendiendo a parámetros como los desarrollados por plataformas como $\mathrm{ARACNE}^{7}$, probablemente los criterios positivos no llegarían ni al 30\%. No en vano, hicimos la prueba con check, el recurso creado por la Red de Humanidades Digitales ${ }^{8} \mathrm{y}$, aunque los resultados obtenidos fueron aceptables ${ }^{9}$, probablemente sea necesaria una evaluación externa por pares algo más rigurosa.

Por otro lado, en la wikivantes no se trabajan bases de datos relacionales, no se utilizan lenguajes de marcado a posteriori tipo XML, ni se utilizan métodos de visualización de la información a partir de algoritmos. El proyecto tampoco tiene el respaldo de una institución académica universitaria ni de un grupo de investigación financiado con fondos públicos o privados y, ni mucho menos, busca ocupar un lugar relevante en el ámbito de las publicaciones científicas, de acceso abierto o cerrado, que posibiliten un mejor posicionamiento de cara a las agencias evaluadoras.

De lo apuntado se infiere, por tanto, que no estamos hablando de un proyecto que se enmarque en el campo de las Humanidades Digitales, ni ahora ni en un futuro próximo, al menos no desde criterios ortodoxos basados en evaluaciones en las que la estadística sea el único instrumento de análisis de los datos. Entre otros motivos, porque además de lo apuntado, falta un componente fundamental en el proceso, el receptor o usuario final, pues como señala acertadamente Elena González-Blanco (2016: 80):

[En las Humanidades Digitales] Las metodologías y formas de trabajo se modifican y pasan de la investigación individual a la colaboración interdisciplinar en equipos, en los que se hace necesaria una estandarización general en varios niveles, y en los que

\footnotetext{
${ }^{7}$ En ARACNE (Red de Humanidades Digitales y Letras Hispánicas) se establecen una serie de pautas para aceptar proyectos de Humanidades Digitales a partir de los siguientes criterios: I. Características (tipo de recurso, diseño conceptual, metainformación); II. Autoría y edición (información, información legal, elementos de calidad); III. Contenidos (materiales propios, conectividad, operatividad); IV. Fiabilidad (indicios de calidad, funcionamiento, productividad externa del recurso); V. Aspectos funcionales (accesibilidad, operatividad, sostenibilidad y permanencia); VI. Requisitos técnicos. (http://ucor1301.cli.lbd.org.es/recursos/criterios).

${ }^{8}$ La herramienta de evaluación se puede consultar en el siguiente enlace del blog de la Red de Humanidades Digitales: http://humanidadesdigitales.net/evaluacion/. Ver Galina (2016: 121-135), al respecto.

9 La evaluación obtuvo unos resultados excelentes en: Información de los responsables, documentación y usabilidad y arquitectura de la información; mientras que requerían atención los apartados sobre Control de calidad, información legal y visibilidad y difusión.
} 
el producto resultante de cada proyecto adquiere diferentes formatos, desde el papel impreso hasta los recursos digitales, bases de datos, plataformas virtuales e infraestructuras digitales. El usuario final cobra importancia en el panorama, convirtiendo la difusión y el medio en un elemento más de la cadena, en el que las redes sociales, las interfaces accesibles o el número de seguidores o colaboradores son un elemento más a tener en cuenta en el planteamiento de cada proyecto conjunto.

Debería entenderse la wikivantes como un puente que se podría tender entre el mundo académico de las Humanidades Digitales (demasiado centrado en ocasiones en la creación de una burbuja que la diferencie de otras disciplinas universitarias) y esa especie de submundo en el que cohabitan los defensores y detractores de la educación y aquellos que abogan por la rentabilidad y enarbolan la bandera de la inutilidad de la Literatura o las Humanidades.

Hoy en día la mayoría de los proyectos utilizan como instrumento y soporte físico lo digital, lo que no supone, necesariamente, que, por sólo este motivo todos los proyectos deban incluirse en el ámbito de las Humanidades Digitales, como es obvio. Pero esta perogrullada tampoco nos debería llevar al extremo opuesto de considerar únicamente aquellos proyectos que cumplan con unas características definidas y cerradas, y unos criterios estrictos regidos por parámetros estadísticos, como pertenecientes al campo de las Humanidades Digitales.

No deberíamos olvidar en ningún momento que los auténticos protagonistas de todo proceso son los materiales, y antes de escoger el instrumento que nos ayude a comprender, analizar y extraer conclusiones críticas en nuestros estudios, deberíamos tener muy claro cómo utilizaremos los recursos y con qué finalidad. Una vez dilucidado esto, deberemos permitir que cada proyecto, digital o no, pueda discurrir, como decía don Quijote al caballero del verde gabán acerca de la querencia de su hijo por la poesía por donde su estrella le llama.

\section{REFERENCIAS BIBLIOGRÁFICAS}

DELORS, J. (1996). La educación encierra un tesoro. Informe a la UNESCO de la comisión internacional sobre la educación para el siglo XXI, presidida por Jacques Delors. Madrid: Santillana, Ediciones UNESCO. Recuperado de http://unesdoc.unesco.org/images/0010/001095/109590so.pdf el 22/02/2017 el 22/02/2017.

GALINA, I. (2016). "La evaluación de los recursos digitales para las Humanidades". Signa. Revista de la Asociación Española de Semiótica, 25, 121-135. 
GARCÍA GUAL, C. (2005). "Apuntes sobre la tradición de la literatura clásica". En Antiquae Lectiones, J. Signes Codoñer, B. Antón Martínez, P. Conde Parrado, M. A. González Manjarrés y J. A. Izquierdo (eds.). Madrid: Cátedra.

GONZÁLEZ, M. (2009). Educar con el Quijote: del canon al hipertexto. Murcia: Ediciones de la Universidad de Murcia.

GONZÁLEZ-BLANCO, E. (2016). "Un nuevo camino hacia las Humanidades Digitales: el Laboratorio de Innovación en Humanidades Digitales de la UNED (LINHD)". Signa. Revista de la Asociación Española de Semiótica, 25, 79-93.

GONZÁLEZ FARACO, J.C. (2010). Lecturas educativas del Quijote. Madrid: Biblioteca Nueva. MARROU, H. (1970). Historia de la educación en la Antigüedad. Buenos Aires: Eudeba.

MEIRIEU, P. (2001). La opción de educar: ética y pedagogía. Barcelona: Octaedro.

NAN-ZHAO, Z. (2005). "Four 'Pillars of Learning' for the Reorientation and Reorganization of Curriculum: Reflections and Discussions". Recuperado de http://unesdoc.unesco.org/images/0010/001095/109590eo.pdf el 22/02/2017.

NUSSBAUM, M. (2005). El cultivo de la humanidad: una defensa clásica de la reforma en la educación liberal. Barcelona: Paidós.

REAL ACADEMIA ESPAÑOLA (2016). Diccionario de la Lengua Española. Edición del Tricentenario. Recuperado de http://dle.rae.es/?w=diccionario el 08/06/2016. (= DRAE) RODRÍGUEZ ORTEGA, N. (2014). "Humanidades Digitales y pensamiento crítico". En Ciencias Sociales y Humanidades Digitales: Técnicas, herramientas y experiencias de e-research e investigación en colaboración, E. Romero Frías y M. Sánchez González (eds.), 13-17. Tenerife: CAC.

SAVATER, F. (2001). El valor de educar. Barcelona: Ariel. 